\title{
Postprandial oxidative stress is exacerbated in cigarette smokers
}

\author{
Richard J. Bloomer*, Adrienne D. Solis, Kelsey H. Fisher-Wellman and Webb A. Smith \\ Department of Health and Sport Sciences, $161 \mathrm{~F}$ Elma Neal Roane Field House, The University of Memphis, Memphis, \\ TN 38152, USA
}

(Received 25 June 2007 - Revised 16 August 2007 - Accepted 10 September 2007 - First published online 10 October 2007)

Both cigarette smoking and high fat meals induce oxidative stress, which is associated with the pathogenesis of numerous diseases. We compared blood antioxidant status, oxidative stress biomarkers and TAG in twenty smokers and twenty non-smokers, matched for age and physical activity, in response to a high fat test meal standardized to body mass. Blood samples were collected before feeding (resting and fasted) and at 1, 2, 4 and $6 \mathrm{~h}$ post feeding and analysed for antioxidant capacity (trolox equivalent antioxidant capacity; TEAC), xanthine oxidase activity (XO), hydrogen peroxide $\left(\mathrm{H}_{2} \mathrm{O}_{2}\right)$, malondialdehyde (MDA) and TAG. Smoking status $(P<0.001)$ and time $(P \leq 0 \cdot 01)$ effects were noted for all variables, with smokers demonstrating higher values compared with non-smokers for all variables except for TEAC, for which values were lower for smokers. $\mathrm{XO}, \mathrm{H}_{2} \mathrm{O}_{2}$, MDA and TAG increased following feeding with a peak response at the $4 \mathrm{~h}$ post feeding time point, with the opposite response occurring for TEAC. Although no interaction effects were noted $(P>0 \cdot 05)$, contrasts revealed greater values in smokers compared with non-smokers for $\mathrm{XO}, \mathrm{H}_{2} \mathrm{O}_{2}$, MDA and TAG, and lower values for TEAC at times from 1-6h post feeding $(P \leq 0.05)$. Our findings indicate that young cigarette smokers experience an exaggerated oxidative stress response to feeding, as well as hypertriacylglycerolaemia, as compared with non-smokers. These data provide insight into another possible mechanism associating cigarette smoking with ill health and disease.

Oxidative stress: Nutrition: Cigarette smoking

Oxidative stress is a condition in which the cellular production of reactive oxygen species (ROS; sometimes referred to as 'free radicals') exceeds the physiological capacity of the antioxidant defence system to render ROS inactive ${ }^{1}$. Increased production of ROS involves the oxidation of lipids and lipoproteins, DNA, proteins and other molecules in ways that impair normal cellular function, possibly resulting in impaired health and disease $\mathrm{e}^{2,3}$. Normal cellular metabolism results in the production of $\operatorname{ROS}^{4}$; however, both physical and environmental stressors can further increase ROS production. In this regard, two primary environmental stressors include cigarette smoking and high fat meals.

Cigarette smoking exacerbates ROS formation and poses a significant oxidant stress in vivo ${ }^{5}$. In one puff of a cigarette, a smoker is exposed to more than $10^{15}$ free radicals in the gas phase alone ${ }^{6}$, with additional exposure in the tar phase equal to more than $10^{17}$ free radicals per g. It has been consistently reported that cigarette smokers have elevated biomarkers of oxidative stress compared with non-smokers ${ }^{7}$ and this represents a potential mechanistic link between regular cigarette smoking and $\mathrm{CVD}^{8}$. The increased oxidative stress observed in smokers may be partly due to the lower blood antioxidant capacity routinely observed in smokers ${ }^{5}$. It is possible that the addition of other ROS generators can further promote oxidative stress in cigarette smokers. Related to this, we have recently reported an exacerbation in physical work-induced oxidative stress in smokers compared with non-smokers 9 .
Aside from cigarette smoking and physical work, numerous studies demonstrate elevated oxidative stress following feeding with high fat meals (for review, see Sies et al. ${ }^{10}$ ). It was first proposed by Zilversmit ${ }^{11}$ that individuals with frequent daily intake of high fat foods exist in a chronic postprandial state, which may be a significant risk factor for atherogenesis. This theory has been developed more recently ${ }^{12}$ and studies have continued to show elevations in oxidative stress biomarkers and TAG during the postprandial state, with a peak occurring between $2-4 \mathrm{~h}$ post feeding ${ }^{13-15}$. Hypertriacylglycerolaemia gives rise to oxidative stress, predisposing to endothelial dysfunction, which is considered an important initial step in the development of atherosclerotic disease under chronic conditions ${ }^{16,17}$. Individuals with elevated resting levels of oxidative stress (i.e. those with metabolic or cardiovascular disorders) have been shown to experience a greater response in oxidative stress biomarkers following intake of standardized meals when compared with healthy control subjects $^{18-21}$. It is possible that cigarette smokers, a population with well-documented elevations in oxidative stress biomarkers $^{22,23}$, may experience a similar response pattern as those with known disease.

To our knowledge, no investigation to date has studied the combined effects of cigarette smoking and feeding on oxidative stress biomarkers. Therefore, in the present investigation we compared blood antioxidant status and oxidative stress biomarkers in smokers and non-smokers in response 
to a high fat test meal standardized to body mass. We were particularly interested in group differences at both the 2 and $4 \mathrm{~h}$ post feeding time points, as these times have been shown to represent the peak in oxidative stress biomarkers in previous studies using non-smokers, with a progressive return towards baseline following $4 \mathrm{~h}$ post feeding. We hypothesized that smokers would have lower antioxidant capacity and higher oxidative stress biomarkers in response to feeding, compared with non-smokers.

\section{Experimental methods}

\section{Participants}

Cigarette smokers ( $n$ 20) and non-cigarette smokers (n 20) met all enrolment criteria and volunteered to participate in the current study. Participants completed a health history and physical activity questionnaire and underwent a physical examination, including anthropometric testing, prior to enrolment. Participants were non-obese (body fat $\leq 25 \%$ for men, $\leq 32 \%$ for women; assessed via seven-site skinfold determination and use of the Seri equation ${ }^{24}$, normolipidaemic (fasting TAG $<150 \mathrm{mg} / \mathrm{dl}$ ) and free of major signs and symptoms suggestive of cardiovascular, metabolic or pulmonary disease as defined by the American College of Sports Medicine $^{25}$. Additionally, participants' self-reports indicated that they did not use medications (e.g. anti-inflammatory or cardiovascular drugs) or nutritional supplements. This was an important consideration, as intake of isolated antioxidant agents could have impacted our outcome measures. Therefore, it was confirmed via both questionnaire and screening interview that no subject in either group used nutritional supplements. Participants needed to regularly smoke five or more cigarettes per $\mathrm{d}$ for a minimum of 6 months immediately preceding the test meal to be enrolled as a smoker. Participants were eligible to be enrolled as non-smokers only if they had not smoked within the past year and were not exposed to passive (second-hand) smoke on a routine basis. These are the same criteria we have used in our previous work $^{9}$. Following the screening procedure, participants were scheduled and given detailed instructions and data forms related to the recording of dietary and physical activity data during the $7 \mathrm{~d}$ before the test meal. All experimental procedures were approved by the University Human Subjects Review Board. Participants provided both verbal and written consent prior to participating. Participant characteristics are presented in Table 1.

\section{Test meal}

All participants reported to the laboratory in the morning (06.00-09.00 hours) following a 10-h overnight fast. Smokers were asked to refrain from smoking for $1 \mathrm{~h}$ prior to reporting. Women reported during the early follicular phase of their menstrual cycle (days $1-5$ ) in order to minimize any potential antioxidant effect of oestrogen, as circulating oestrogen levels are lowest during this time. Following a 10 min quiet rest period, a pre-meal blood sample was collected. Participants then consumed the test meal (within $15 \mathrm{~min}$ ), which consisted of a milkshake made with a combination of whole milk, ice cream and whipping cream. The size of the milkshake was
Table 1. Descriptive characteristics of smokers and non-smokers§ (Mean values and standard deviations)

\begin{tabular}{|c|c|c|c|c|}
\hline \multirow[b]{2}{*}{ Variable } & \multicolumn{2}{|c|}{ Smokers $†$} & \multicolumn{2}{|c|}{ Non-smokers $\ddagger$} \\
\hline & Mean & SD & Mean & SD \\
\hline Age (years) & 23 & 6 & 23 & 4 \\
\hline Height $(\mathrm{cm})$ & 174 & 10 & 168 & 8 \\
\hline Weight (kg) & 75 & 16 & 69 & 14 \\
\hline BMI $\left(\mathrm{kg} / \mathrm{m}^{2}\right)$ & $24 \cdot 6$ & 3 & $24 \cdot 4$ & 4 \\
\hline Body fat (\%) & 18 & 5 & 19 & 6 \\
\hline Waist:hip & 0.79 & 0.06 & 0.74 & 0.06 \\
\hline Resting heart rate (bpm) & 67 & 11 & 67 & 11 \\
\hline Resting SBP (mmHg) & 113 & 10 & 117 & 12 \\
\hline Resting DBP (mmHg) & 72 & 8 & 72 & 8 \\
\hline Total cholesterol (mg/dl)* & 181 & 20 & 157 & 24 \\
\hline HDL-cholesterol (mg/dl) & 47 & 11 & 49 & 16 \\
\hline LDL-cholesterol (mg/dl) & 111 & 31 & 91 & 36 \\
\hline Total cholesterol:HDL & 3.8 & $1 \cdot 1$ & $3 \cdot 1$ & $1 \cdot 1$ \\
\hline Cigarettes per d & 9 & 5 & NA & NA \\
\hline Range & $5-20$ & & & \\
\hline Years smoking & 5 & 3 & NA & NA \\
\hline Range & $1-10$ & & & \\
\hline Pack years & 2 & 2 & NA & NA \\
\hline Range & $0.125-6.75$ & & & \\
\hline
\end{tabular}

$\mathrm{SBP}$, systolic blood pressure; DBP, diastolic blood pressure; bpm, beats per min; NA, not applicable.

* No statistical differences were noted between groups for any above variable $(P>0.05)$ with the exception of total cholesterol $(P=0.04)$.

†Smokers are eleven men, nine women.

† Non-smokers are nine men, eleven women.

$\S$ For details of subjects and procedures, see Experimental methods.

based on participants' body mass and equivalent to $1.2 \mathrm{~g}$ fat and carbohydrate and $0.25 \mathrm{~g}$ protein per $\mathrm{kg}$. The shake provided approximately $71 \mathrm{~kJ}(17 \mathrm{kcal})$ per $\mathrm{kg}$. These parameters were based on previous studies in which test meals have included fat content between $1.0-1.4 \mathrm{~g} / \mathrm{kg}$ body mass, carbohydrate content between $0.9-1 \cdot 3 \mathrm{~g} / \mathrm{kg}$ body mass and protein content between $0 \cdot 2-0 \cdot 4 \mathrm{~g} / \mathrm{kg}$ body mass. The postprandial observation period lasted $6 \mathrm{~h}$ (from the start of meal intake), during which time four additional blood samples were collected $(1,2,4$ and $6 \mathrm{~h}$ post meal). Participants remained in the laboratory during this period and expended as little energy as possible (i.e. watched movies, worked on the computer). No additional meals or energy-containing beverages were allowed during this period and smokers refrained from smoking cigarettes. Water was allowed ad libitum and the majority of subjects consumed between 16 and 24 oz during the postprandial period.

\section{Blood sampling and biochemistry}

Venous blood samples (approximately $20 \mathrm{ml}$ ) were taken from participants' forearms via needle and vacutainer using minimal stasis pre-meal (resting and fasting; $0 \mathrm{~h}$ ) and at 1, 2, 4 and $6 \mathrm{~h}$ post meal. Blood was processed immediately and stored in multiple aliquots at $-80^{\circ} \mathrm{C}$ until analysed. All assays were performed in duplicate and on first thaw. Antioxidant capacity was measured in serum using the Trolox-equivalent antioxidant capacity assay using procedures outlined by the reagent provider (Sigma Chemical) and as previously described $^{26}$. Xanthine oxidase activity and hydrogen peroxide were both measured in plasma using the Amplex Red reagent 
method as described by the manufacturer (Molecular Probes, Invitrogen Detection Technologies). Malondialdehyde was measured in plasma using commercially available reagents (Northwest Life Science Specialties), using the method described by Jentzsch et al. ${ }^{27}$. Assays for TAG, glucose, total cholesterol and HDL-cholesterol were performed using serum following standard enzymatic procedures as described by the reagent manufacturer (Thermo Electron Clinical Chemistry). Prior to HDL-cholesterol analysis, precipitation of apo B containing lipoproteins (VLDL, LDL and Lp(a)) was performed using phosphotungstic acid, coupled with centrifugation, and HDL-cholesterol was measured in the supernatant. LDL-cholesterol was calculated using the Friedwald equation as follows:

$$
\begin{aligned}
\text { LDL-cholesterol } & =\text { total cholesterol }- \text { HDL-cholesterol } \\
& -(\mathrm{TAG} / 5)
\end{aligned}
$$

\section{Dietary and physical activity records}

All participants were instructed to maintain their normal diet and activity and to record these variables on $\log$ forms during the $7 \mathrm{~d}$ period prior to the assigned test day. Nutritional records were analysed for total dietary energy, protein, carbohydrate, fat and a variety of antioxidant micronutrients (Food Processor SQL, version 9.9; ESHA Research). Activity logs were reviewed to determine the amount of activity done during the week prior to the test day. Subjects were given specific instructions to avoid physically stressful tasks (including exercise) during the $24 \mathrm{~h}$ period preceding the test meal. This was important in order to control for any acute effects of physical activity on postprandial oxidative stress, as a recent study has demonstrated that acute strenuous exercise may attenuate the rise in oxidative stress observed following feeding ${ }^{14}$.

\section{Statistical analysis}

All outcome variables were analysed using a 2 (group) $\times 5$ (time) repeated measures ANOVA. Contrasts were performed for comparison of smokers and non-smokers at all times. Pairwise correlations were made between all outcome variables. The outcome data are presented as means with their standard errors of the mean. Dietary, physical activity and descriptive data were analysed using a one way ANOVA and presented as means and standard deviations. All analyses were performed using JMP statistical software (version 4.0.3; SAS Institute). Statistical significance was set at $P \leq 0.05$.

\section{Results}

Regarding descriptive characteristics, smokers and non-smokers were not different $(P>0.05)$, with the exception of smokers having higher total cholesterol compared with non-smokers $(P=0.04$; Table 1$)$. With regard to dietary intake, although smokers consumed less total daily energy (8062 (SD 1557) v. 9954 (SD 1762) kJ; 1926 (SD 372) v. 2378 (SD 421) kcal), protein (80 (SD 19) v. 106 (SD 18) g), carbohydrate (232 (SD 64) v. 287 (SD 55) g), fat (66 (SD 21) v. 77 (SD 18) g), $\beta$-carotene (5.1 (SD 3.2) v. 8.3
(SD 3.7) $\mathrm{mg}$ ) and Se (40 (SD 14) v. 57 (SD 18) $\mu \mathrm{g}$ ) compared with non-smokers, these variables were not statistically different $(P>0.05)$. However, smokers consumed statistically less vitamin C (45 (SD 26) v. 128 (SD 42) $\mathrm{mg} ; P=0.03$ ) and vitamin E (4 (SD 2) v. 10 (SD 3) $\mathrm{mg} ; P=0.01$ ) compared with non-smokers. This is not surprising, as previous studies have indicated lower antioxidant nutrient intake for smokers compared with non-smokers ${ }^{28}$, largely due to lower fruit and vegetable consumption by smokers ${ }^{29,30}$. We noted this to be the case in our sample of smokers as well. That is, few fruits and vegetables were consumed during the $7 \mathrm{~d}$ diet reporting period, with the majority of dietary energy consumed in the form of processed foods, which traditionally are lower in antioxidant nutrients. The total amount of moderate to strenuous habitual physical activity performed by smokers and non-smokers based on selfreport did not differ (3.5 (SD 2) v. 3.6 (SD 2) h per week), and was mirrored during the week prior to the test meal.

No baseline (pre-meal) differences were noted between smokers and non-smokers for any outcome variable $(P>0 \cdot 05)$. Both smoking status $(P=0.0002)$ and time $(P=0.01)$ main effects were noted for antioxidant capacity, and contrasts at the 1 $(P=0.05), 2(P=0.024)$ and $4(P=0.015) \mathrm{h}$ post feeding time points revealed lower values in smokers compared with nonsmokers (Fig. 1). In relation to xanthine oxidase (Fig. 2), malondialdehyde (Fig. 3) and TAG (Fig. 4), both smoking status $(P<0.0001)$ and time $(P<0.0001)$ main effects were noted. The same was true for hydrogen peroxide (Fig. 5), although the level of significance was different for the smoking status main effect $(P=0 \cdot 0002)$. For all of these variables, smokers demonstrated higher values compared with non-smokers, and values increased following feeding, with a peak response observed at the $4 \mathrm{~h}$ post feeding time point. Although no interaction effects were noted $(P>0.05)$, group differences were apparent for xanthine oxidase at $2(P=0.05), 4$ $(P=0.003)$ and $6(P=0.03) \mathrm{h}$ post feeding; for hydrogen peroxide at $2(P=0.05)$ and $4(P=0.0005) \mathrm{h}$ post feeding; for

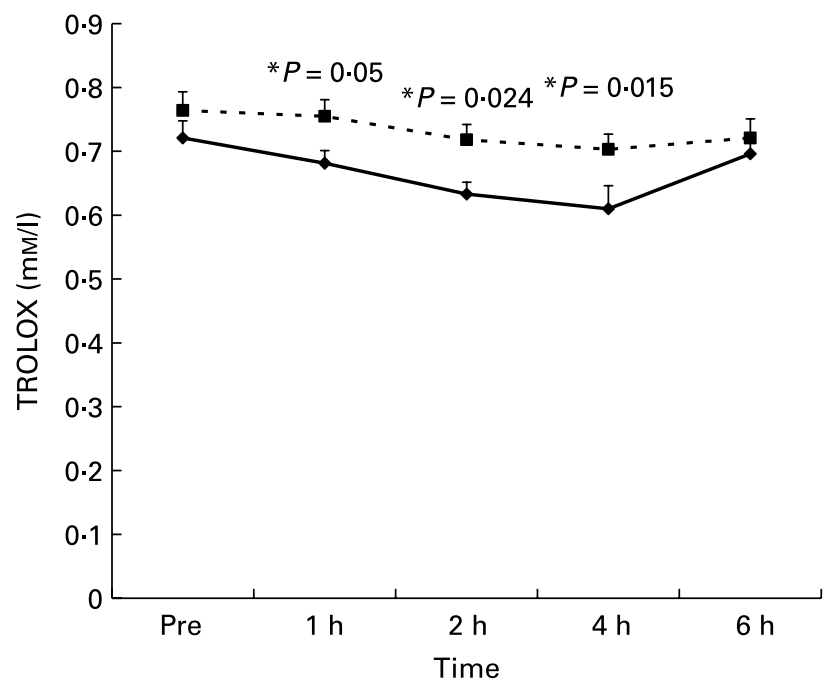

Fig. 1. Serum trolox equivalent antioxidant capacity $(\mathrm{mm} / \mathrm{l})$ before and following intake of a high fat meal in smokers $(--)$ and non-smokers $(-\mathbf{-}-)$. Values are means, with their standard errors represented by vertical bars. Smoking status $(P=0.0002)$ and time $(P=0.01)$ main effects; * Significant differences between smokers and non-smokers using planned contrasts. For details of subjects and procedures, see Experimental methods. 


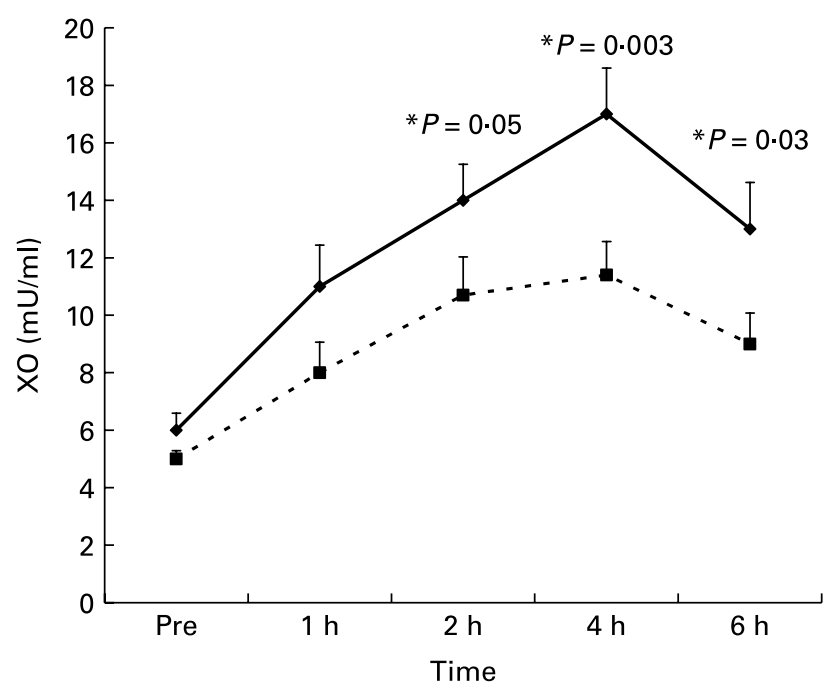

Fig. 2. Plasma xanthine oxidase $(\mathrm{XO})$ activity $(\mathrm{mU} / \mathrm{ml})$ before and following intake of a high fat meal in smokers $(--)$ and non-smokers $(-\square-)$. Values are means, with their standard errors represented by vertical bars. Smoking status $(P<0.0001)$ and time $(P<0.0001)$ main effects; * Significant differences between smokers and non-smokers using planned contrasts. For details of subjects and procedures, see Experimental methods.

malondialdehyde at $1(P=0.03), 2(P=0.007), 4(P=0.0004)$ and $6(P=0 \cdot 01) \mathrm{h}$ post feeding; for TAG at $1(P=0.007), 2$ $(P=0.001)$ and $4(P=0 \cdot 0003)$ h post feeding. Not surprisingly, these four variables were positively correlated with one another $(r 0.33-0.73 ; P<0.00001)$ and negatively correlated to antioxidant capacity $(r 0 \cdot 20-0 \cdot 46 ; P \leq 0 \cdot 003)$. A smoking status main effect was noted for glucose, with smokers having higher values compared with non-smokers $(P=0.001)$, with fasting values for smokers and non-smokers equal to 91 (SE 5) $\mathrm{mg} / \mathrm{dl}$ and 82 (SE 4) $\mathrm{mg} / \mathrm{dl}$, respectively. No other effects were noted for glucose $(P>0.05)$ and values were relatively unchanged (i.e. increased $5-8 \mathrm{mg} / \mathrm{dl}$ ) over time for both smokers and non-smokers (data not shown).

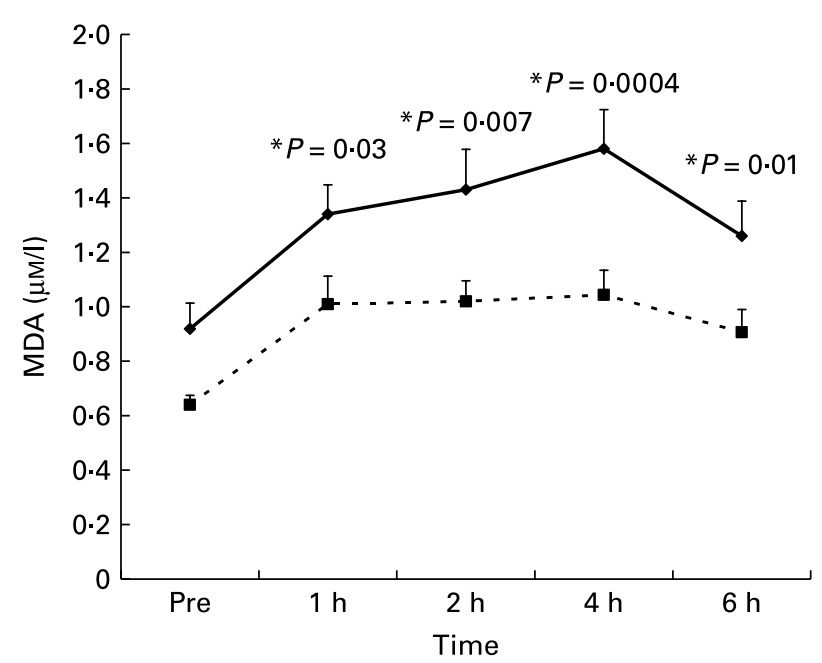

Fig. 3. Plasma malondialdehyde (MDA; $\mu \mathrm{M} / \mathrm{l})$ before and following intake of a high fat meal in smokers $(--)$ ) and non-smokers $(-\mathbf{-}-)$. Values are means, with their standard errors represented by vertical bars. Smoking status $(P<0.0001)$ and time $(P<0.0001)$ main effects; * Significant differences between smokers and non-smokers using planned contrasts. For details of subjects and procedures, see Experimental methods.

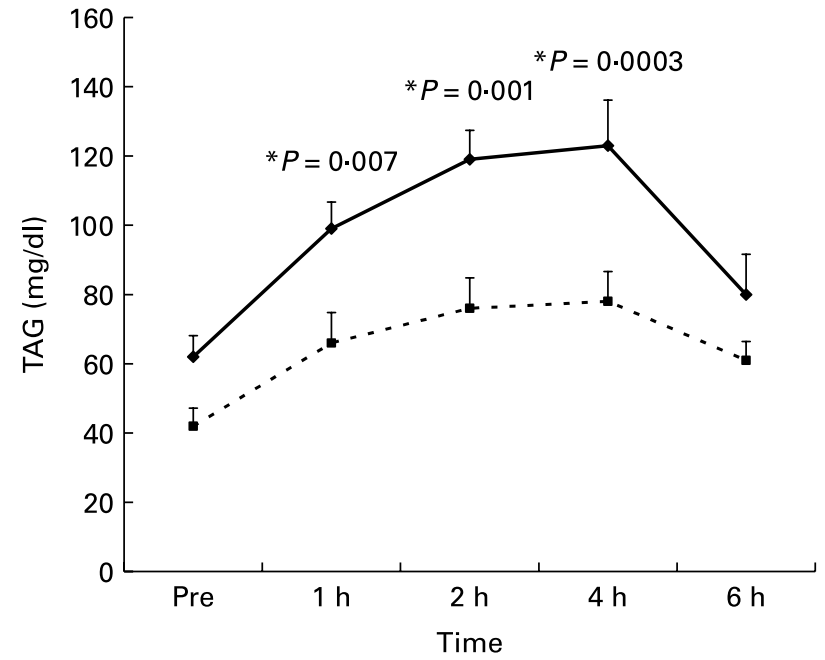

Fig. 4. Serum TAG (mg/dl) before and following intake of a high fat meal in smokers (- -$)$ and non-smokers (- - $)$. Values are means, with their standard errors represented by vertical bars. Smoking status $(P<0.0001)$ and time $(P<0.0001)$ main effects; * Significant differences between smokers and non-smokers using planned contrasts. For details of subjects and procedures, see Experimental methods.

\section{Discussion}

Data from the present investigation indicate that cigarette smokers experience an exaggerated oxidative stress and TAG response to a high fat meal when compared with agematched non-smokers. This is the first study to our knowledge to report postprandial oxidative stress and TAG data in reference to a population of cigarette smokers. The present findings provide additional insight into another possible mechanism associating cigarette smoking with ill health and disease. It should be noted that these data were obtained using a very young population of novice smokers (e.g. $23 \pm 5$ years;

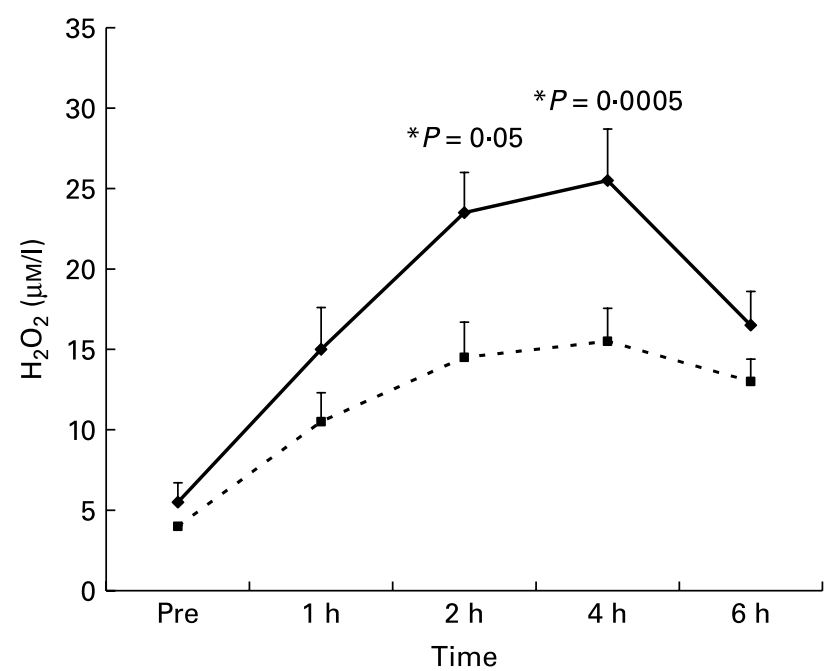

Fig. 5. Plasma hydrogen peroxide $\left(\mathrm{H}_{2} \mathrm{O}_{2} ; \mu \mathrm{M} / \mathrm{l}\right)$ before and following intake of a high fat meal in smokers $(--)$ ) and non-smokers $(-\boldsymbol{-}-)$. Values are means, with their standard errors represented by vertical bars. Smoking status $(P=0.0002)$ and time $(P<0.0001)$ main effects; * Significant differences between smokers and non-smokers using planned contrasts. For details of subjects and procedures, see Experimental methods. 
$2 \pm 2$ pack years). It is possible that older, more established smokers may experience a more pronounced oxidative stress response to feeding.

The smokers and non-smokers in the present study were of similar age and performed low amounts of physical activity (3.5 (SD 2) v. 3.6 (SD 2) h per week for smokers and non-smokers, respectively). With regard to dietary intake, significant differences were only noted between groups for vitamins $\mathrm{C}$ and $\mathrm{E}$, with smokers having lower intakes of both vitamins. It is certainly possible that this could have contributed to the increased oxidative stress response following feeding, as both the antioxidant capacity of blood ${ }^{31}$ as well oxidative stress biomarkers ${ }^{32}$ can be influenced by nutrient intake. While it is likely that the act of smoking is most responsible for findings of increased oxidative stress, as demonstrated recently when controlling for nutrient intake ${ }^{33}$, it is unknown what the independent effect of lower dietary antioxidant intake in our sample of smokers was on the measured biomarkers. Therefore, we must consider the possibility that the current results are due in part to the combination of chronic cigarette smoking coupled with a diet deficient in antioxidants. Future studies in this area may attempt to better equate exogenous antioxidant intake between smokers and non-smokers (possibly by providing standardized meals and/or supplemental antioxidants to subjects in both groups) in order to more specifically address the independent effects of chronic smoking on postprandial oxidative stress.

Smokers have been shown to possess lower fasting blood antioxidant capacity compared with non-smokers ${ }^{5}$ due in part to both a lower dietary antioxidant intake and a depletion of blood antioxidants due to routine exposure to ROS from cigarette smoke. We hypothesized that blood antioxidant capacity would also be lower following feeding in smokers compared with non-smokers, due to the increased consumption of blood antioxidants in an effort to 'protect' macromolecules from oxidation as they are exposed to high amounts of ROS generated from the test meal. While both groups experienced a decrease in antioxidant capacity, smokers demonstrated a more pronounced decline at the 1,2 and $4 \mathrm{~h}$ post feeding time points (Fig. 1).

As mentioned earlier, antioxidant capacity in both groups declined following feeding, likely due to the consumption of blood antioxidants to counteract the increase in ROS production with feeding. We chose to measure xanthine oxidase activity as a surrogate marker of ROS production, as well as hydrogen peroxide, as both of these biomarkers are highly related and provide a good overall representation of biological oxidative stress. Xanthine oxidase is commonly used as a marker of oxidative stress ${ }^{34}$ as this enzyme is a ubiquitous generator of radical species, ultimately leading to superoxide radical. In aqueous solutions, the superoxide radical is poorly reactive; however, it can be readily converted into hydrogen peroxide, through the activity of superoxide dismu$\operatorname{tase}^{35}$. As with superoxide radical, much of the cytotoxic effects of hydrogen peroxide are due to its conversion into the hydroxyl radical, the most reactive oxygen-containing species known ${ }^{3}$. This conversion can occur in the presence of reduced transition metals such as $\mathrm{Cu}$ and $\mathrm{Fe}$ via the Fenton reaction ${ }^{36}$ or by the interaction of superoxide with hydrogen peroxide through the Haber-Weiss reaction ${ }^{37}$. With a rise in hydroxyl radicals, the potential exists for damage to various cellular structures, including lipids ${ }^{38}$. Malondialdehyde is a representation of ROS oxidation of lipids, involving degradation of PUFA and phospholipids through a chain reaction sequence ${ }^{38}$, typically initiated by a hydroxyl radical.

In regard to the afore-mentioned three variables, we noted a greater response for xanthine oxidase activity (Fig. 2), malondialdehyde (Fig. 3) and hydrogen peroxide (Fig. 5) for smokers compared with non-smokers following feeding. In previous studies, it has been noted that individuals with certain diseases experience a more robust increase in oxidative stress compared with healthy control subjects. For example, in postprandial studies involving high fat test meals, diabetics experienced higher oxidative stress levels than healthy controls ${ }^{18,21}$. The same is true with postprandial oxidative stress in patients with $\mathrm{CVD}^{19}$. It has recently been suggested that obesity ${ }^{20}$ and ageing ${ }^{39}$ will promote greater postprandial oxidative stress. It should be noted that all studies examining postprandial oxidative stress in diseased subjects also report an increase in oxidative stress in healthy control subjects, albeit to a lesser extent.

Data from the present study indicate that cigarette smokers experience an exaggerated oxidative stress response to feeding, in a similar manner as patients with known disease. While this is the first study to report such findings, the mechanism(s) responsible for these changes remains to be elucidated. Future study is needed in this area.

In support of our findings for greater lipid peroxidation (i.e. malondialdehyde) in smokers compared with non-smokers, we noted a similar increase in TAG. Coupled with the lower blood antioxidant capacity, the increase in blood TAG following feeding provides greater opportunity for lipid peroxidation and, hence, the formation of malondialdehyde. These findings agree with previous work demonstrating a peak TAG response at $4 \mathrm{~h}$ post feeding, with a similar corresponding increase in lipid peroxidation ${ }^{14}$. While not a primary objective of the current study, we measured blood glucose in response to feedings and noted minimal change in values over time for both smokers and non-smokers. This is likely due to both the time course of our measurements (i.e. first measurement post feeding not occurring until $1 \mathrm{~h}$ ), as well as the inclusion of a high amount of dietary fat to the test meal. Almost identical findings have been reported recently by Blendea and colleagues ${ }^{13}$.

In conclusion, we report for the first time that cigarette smokers experience an exaggerated oxidative stress and TAG response to a standardized high fat meal when compared with age-matched non-smokers. These findings add to the already well-documented elevation in fasting oxidative stress levels in chronic cigarette smokers. Therefore, smokers who typically consume frequent high fat meals may be at an even greater risk of oxidative stress-related disease.

\section{Acknowledgements}

Funding for this work was provided by The University of Memphis. The authors declare that they have no competing interests.

\section{References}

1. Bloomer RJ \& Goldfarb AH (2004) Anaerobic exercise and oxidative stress: a review. Can J Appl Physiol 29, 245-263. 
2. Chakravati B \& Chakravati DN (2007) Oxidative modification of proteins: Age-related changes. Gerontology 53, 128-139.

3. Halliwell B (1984) Oxygen radicals: a commonsense look at their nature and medical importance. Med Biol 62, 71-77.

4. Asmus K \& Bonifacic M (2000) Free radical chemistry. In Handbook of Oxidants and Antioxidants in Exercise, pp. 3-54 [CK Sen, L Packer and O Hanninen, editors]. Amsterdam, The Netherlands: Elsevier.

5. Alberg AJ (2002) The influence of cigarette smoking on circulating concentrations of antioxidant micronutrients. Toxicology 180, $121-137$.

6. Pryor WA \& Stone K (1993) Oxidants in cigarette smoke. Radicals, hydrogen peroxide, peroxynitrate, and peroxynitrite. Ann N Y Acad Sci 686, 12-27.

7. Burke A \& FitzGerald GA (2003) Oxidative stress and smoking-induced vascular injury. Prog Cardiovasc Dis 46, 79-90.

8. Ambrose JA \& Barua RS (2004) The pathophysiology of cigarette smoking and cardiovascular disease: An update. J Am Coll Cardiol 43, 1731-1737.

9. Bloomer RJ, Creasy AK \& Smith WA (2007) Physical workinduced oxidative stress is exacerbated in young cigarette smokers. Nicotine Tob Res 9, 205-211.

10. Sies H, Stahl W \& Sevanian A (2005) Nutritional, dietary and postprandial oxidative stress. J Nut 135, 969-972.

11. Zilversmit DB (1979) Atherogenesis: A postprandial phenomenon. Circulation 60, 473-485.

12. Ceriello A, Taboga C, Tonutti L, Quagliaro L, Piconi L, Bais B, Da Ros R \& Motz E (2002) Evidence for an independent and cumulative effect of postprandial hypertriglyceridemia and hyperglycemia on endothelial dysfunction and oxidative stress generation: effects of short- and long-term simvastatin treatment. Circulation 106, 1211-1218.

13. Blendea MC, Bard M, Sowers JR \& Winer N (2005) High-fat meal impairs vascular compliance in a subgroup of young healthy subjects. Metabolism 54, 1337-1344.

14. McClean CM, McLaughlin J, Burke G, Murphy MH, Trinick T, Duly E \& Davison GW (2007) The effect of acute aerobic exercise on pulse wave velocity and oxidative stress following postprandial hypertriglyceridemia in healthy men. Eur J Appl Physiol 100, 225-234

15. Tsai WC, Li YH, Lin CC, Chao TH \& Chen JH (2004) Effects of oxidative stress on endothelial function after a high-fat meal. Clin Sci 106, 315-319.

16. Bae JH, Bassenge E, Kim KB, Kim YN, Kim KS, Lee HJ, Moon KC, Lee MS, Park KY \& Schwemmer M (2001) Postprandial hypertriglyceridemia impairs endothelial function by enhanced oxidant stress. Atherosclerosis 155, 517-523.

17. de Koning EJ \& Rabelink TJ (2002) Endothelial function in the post-prandial state. Atheroscler Suppl 3, 11-16.

18. Dierckx N, Horvath G, van Gils C, Vertommen J, van de Vliet J, DeLeeuw I \& Manuel-y-Keenoy B (2003) Oxidative stress status in patients with diabetes mellitus: Relationship to diet. Eur J Clin Nutr 57, 999-1008.

19. Graner M, Kahri J, Nakano T, Sarna SJ, Nieminen MS, Syvanne M \& Taskinen MR (2006) Impact of postprandial lipaemia on low-density lipoprotein (LDL) size and oxidized LDL in patients with coronary artery disease. Eur J Clin Invest 36, 764-770.

20. Rask-Madsen C \& King GL (2007) Mechanisms of disease: Endothelial dysfunction in insulin resistance and diabetes. Nat Clin Pract Endocrinol Metab 3, 46-56.
21. Saxena R, Madhu SV, Shukla R, Prabhu KM \& Gambhir JK (2005) Postprandial hypertriglyceridemia and oxidative stress in patients of type 2 diabetes mellitus with macrovascular complications. Clin Chim Acta 359, 101-108.

22. Lodovici M, Casalini C, Cariaggi R, Michelucci L \& Dolara P (2000) Levels of 8-hydroxydeoxyguanosine as a marker of DNA damage in human leukocytes. Free Radic Biol Med 28, 13-17.

23. Morrow JD, Frei B, Longmire AW, Gaziano MJ, Lynch SM, Shyr Y, Strauss WE, Oates JA \& Roberts LJ (1995) Increase in circulating products of lipid peroxidation $\left(\mathrm{F}_{2}\right.$-isoprostanes $)$ in smokers. New Eng J Med 332, 1198-1203.

24. Jackson AS \& Pollock ML (1985) Practical assessment of body composition. Phys Sport Med 13, 76-90.

25. Whaley MH (editor) (2000) ACSM's Guidelines for Exercise Testing and Prescription, 7th ed. Philadelphia: Lippincott Williams \& Wilkins.

26. Rice-Evans CA (2000) Measurement of total antioxidant activity as a marker of antioxidant status in vivo: procedures and limitations. Free Rad Res 33, Suppl., S59-S66.

27. Jentzsch AM, Bachmann H, Furst P \& Biesalski HK (1996) Improved analysis of malondialdehyde in human body fluids. Free Rad Biol Med 20, 251-256.

28. Ma J, Hampl JS \& Betts NM (1994-1996) Antioxidant intakes and smoking status: data from the continuing survey of food intakes by individuals. Am J Clin Nutr 71, 774-780.

29. Baer Wilson D \& Nietert PJ (2002) Patterns of fruit, vegetable, and milk consumption among smoking and non-smoking female teens. Am J Prev Med 22, 240-246.

30. Wilson DB, Smith BN, Speizer IS, Bean MK, Mitchell KS, Uguy LS \& Fries EA (2005) Differences in food intake and exercise by smoking status in adolescents. Prev Med 40, 872-879.

31. Cao G, Booth SL, Sadowski JA \& Prior RL (1998) Increases in human plasma antioxidant capacity after consumption of controlled diets high in fruit and vegetables. Am J Clin Nutr $\mathbf{6 8}$, $1081-1087$.

32. Watson TA, Callister R, Taylor RD, Sibbritt DW, MacDonaldWicks LK \& Garg ML (2005) Antioxidant restriction and oxidative stress in short-duration exhaustive exercise. Med Sci Sports Exerc 37, 63-71.

33. Northrop-Clewes CA \& Thurnham DI (2007) Monitoring micronutrients in cigarette smokers. Clin Chim Acta 377, $14-38$.

34. Hellsten Y (2000) The role of xanthine oxidase in exercise. In Handbook of Oxidants and Antioxidants in Exercise, 1st ed., pp. 153-176 [CK Sen, L Packer and O Hanninen, editors]. Amsterdam, The Netherlands: Elsevier.

35. McCord JM \& Fridovich I (1969) Superoxide dismutase. An enzymic function for erythrocuprein (hemocuprein). $J$ Biol Chem 244, 6049-6055.

36. Wardman P \& Candeias LP (1996) Fenton chemistry: an introduction. Radiat Res 145, 523-531.

37. Liochev SI \& Fridovich I (2002) The Haber-Weiss cycle - 70 years later: an alternative view. Redox Rep 7, 55-57.

38. Alessio HM (2000) Lipid peroxidation in healthy and diseased models: Influence of different types of exercise. In Handbook of Oxidants and Antioxidants in Exercise, pp. 3-54 [CK Sen, L Packer and O Hanninen, editors]. Amsterdam, The Netherlands: Elsevier.

39. Droge W (2005) Oxidative stress and ageing: Is ageing a cysteine deficiency syndrome? Philos Trans $R$ Soc Lond B Biol Sci 360, 2355-2372. 